\title{
Intravoxel Incoherent Motion MR Imaging in the Differentiation of Benign and Malignant Sinonasal Lesions: Comparison with Conventional Diffusion-Weighted MR Imaging
}

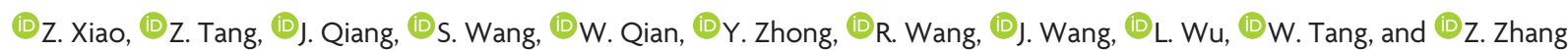

\begin{abstract}
BACKGROUND AND PURPOSE: Intravoxel incoherent motion is a promising method for the differentiation of sinonasal lesions. This study aimed to evaluate the value of intravoxel incoherent motion in the differentiation of benign and malignant sinonasal lesions and to compare the diagnostic performance of intravoxel incoherent motion with that of conventional DWI.
\end{abstract}

MATERIALS AND METHODS: One hundred thirty-one patients with histologically proved solid sinonasal lesions (56 benign and 75 malignant) who underwent conventional DWI and intravoxel incoherent motion were recruited in this study. The diffusion coefficient (D), pseudodiffusion coefficient $\left(D^{*}\right)$, and perfusion fraction $(f)$ values derived from intravoxel incoherent motion and ADC values derived from conventional DWI were measured and compared between the 2 groups using the Student $t$ test. Receiver operating characteristic curve analysis, logistic regression analysis, and 10-fold cross-validation were performed to evaluate the diagnostic performance of singleparametric and multiparametric models.

RESULTS: The mean ADC and $D$ values were significantly lower in malignant sinonasal lesions than in benign sinonasal lesions (both $P<$ .001). The mean $f$ value was higher in malignant lesions than in benign lesions ( $P=.003)$. Multiparametric models can significantly improve the cross-validated areas under the curve for the differentiation of sinonasal lesions compared with single-parametric models (all corrected $P<.05$ except the $D$ value). The model of $D+f$ provided a better diagnostic performance than the ADC value (corrected $P<.001$ ).

CONCLUSIONS: Intravoxel incoherent motion appears to be a more effective MR imaging technique than conventional DWI in the differentiation of benign and malignant sinonasal lesions.

ABBREVIATIONS: AUC = area under the curve; $D=$ diffusion coefficient; $D^{*}=$ pseudodiffusion coefficient; $f$ = perfusion fraction; IVIM = intravoxel incoherent motion; $R O C=$ receiver operating characteristic.

$\mathbf{T}$ he sinonasal area may contain a wide spectrum of benign and malignant tumors and tumorlike lesions of epithelial, mesenchymal, neural/neuroectodermal, or hematopoietic or-

Received August 17, 2017; accepted after revision November 14

From the Departments of Radiology (Z.X., Z.T., W.Q., R.W.), Pathology (S.W.), Radiotherapy (J.W.), and Otolaryngology (L.W.), Eye \& ENT Hospital of Shanghai Medical School, Fudan University, Shanghai, P. R. China; Department of Radiology (J.Q., Y.Z.), Jinshan Hospital of Shanghai Medical School, Fudan University, Shanghai, P. R. China; and Siemens Healthcare Ltd (W.T., Z.Z.), Shanghai, P. R. China.

Zebin Xiao, Zuohua Tang, and Jinwei Qiang contributed equally to this study.

This work was supported by the Grant of Science and Technology Commission of Shanghai Municipality (No. 17411962100; 14411962000) and Shanghai Municipal Commission of Health and Family Planning (Grant No. ZK2015A05).

Please address correspondence to Zuohua Tang, MD, PhD, Department of Radiology, Eye \& ENT Hospital of Shanghai Medical School, Fudan University, 83 Fenyang Rd, Shanghai, 200031, P. R. China; e-mail: tzh518sunny@163.com; Shuyi Wang, MD, Department of Pathology, Eye \& ENT Hospital of Shanghai Medical School, Fudan University, 83 Fenyang Rd, Shanghai, 200031, P. R. China; e-mail: wangshuyi@eent.shmu.edu.cnl

- Indicates open access to non-subscribers at www.ajnr.org

http://dx.doi.org/10.3174/ajnr.A5532 igins, ${ }^{1}$ composing approximately $3 \%$ of all head and neck tumors. ${ }^{2-4}$ The clinical presentations of sinonasal lesions are often nonspecific. ${ }^{5}$ Patients with malignant sinonasal tumors often have an overall poor prognosis and generally require radiation therapy and chemotherapy as well as surgical resection, ${ }^{6}$ whereas patients with benign lesions, such as polyps, often undergo complete surgical resection or clinical follow-up without surgery. ${ }^{7}$ Thus, the differentiation of benign and malignant sinonasal lesions is of great importance for therapeutic decisions and prognosis. Cross-sectional imaging techniques, such as CT and MR imaging, play an important role in the differentiation of sinonasal lesions. Nevertheless, the morphologic imaging features of benign and malignant sinonasal lesions are often nonspecific and overlap. ${ }^{4,8,9}$

Conventional diffusion-weighted MR imaging with the measurement of apparent diffusion coefficient values may aid in the discrimination of malignant and benign lesions in the head and neck and the sinonasal area. ${ }^{9-12}$ However, some overlap of ADC values was also observed. ${ }^{10,13-15}$ It has been docu- 
mented that microvascular flow through the complex capillary network generates a "pseudodiffusion" effect known as intravoxel incoherent motion (IVIM). ${ }^{13,14}$ According to IVIM analysis, both tissue diffusivity and microcapillary perfusion can be quantified separately by fitting the MR signal acquired at different b-values to a biexponential model. ${ }^{15}$ Consequently, 3 parameters can be obtained from IVIM, including $D, D^{*}$, and $f$, which represent the pure diffusion coefficient, pseudodiffusion coefficient, and perfusion fraction, respectively. Recently, IVIM MR imaging has emerged as a potential new method for the differentiation of head and neck tumors and the prediction of tumor responses to treatment. ${ }^{16-22}$

However, to the best of our knowledge, no studies in differentiating benign and malignant sinonasal lesions using IVIM have been performed. In this study, we aimed to investigate IVIM as a tool for distinguishing benign and malignant sinonasal lesions and to compare the differential diagnostic performance of IVIM with conventional DWI in sinonasal lesions.

\section{MATERIALS AND METHODS \\ Study Population}

The institutional review board of Eye \& ENT Hospital of Fudan University approved this retrospective study, and the requirement for patient informed consent was waived due to the retrospective nature of this study. One hundred thirty-one consecutive patients with sinonasal solid masses were recruited from May 2015 to March 2017. All masses were confirmed by surgery/biopsy and histopathology. Patients who previously had a history of treatment or recurrence were excluded.

\section{MR Imaging Techniques}

MR imaging examinations were performed on a 3T MR imaging scanner (Magnetom Verio; Siemens, Erlangen, Germany) using a 12-channel head coil. Conventional MR images were obtained; then DWI was performed using a single-shot echo-planar imaging sequence with a bipolar scheme along all 3 orthogonal axes to obtain isotropic DWI. Imaging parameters were as follows: TR/ $\mathrm{TE}=5200 / 83 \mathrm{~ms}$, number of averages $=2$, acquisition matrix $=$ $120 \times 120, \mathrm{FOV}=230 \mathrm{~mm}$, slices $=5$, slice thickness $=5 \mathrm{~mm}$, parallel imaging acceleration factor $=2$. Eleven different $b$ factors ranging from 0 to $1000 \mathrm{~s} / \mathrm{mm}^{2}$ were used $(b=0,50,100,150,200$, $250,300,350,400,800$, and $\left.1000 \mathrm{~s} / \mathrm{mm}^{2}\right)$. The total scan time was 6 minutes 39 seconds.

\section{Image Processing and Analysis}

The conventional monoexponential DWI model is mathematically expressed by the equation $S_{\mathrm{b}} / S_{0}=\exp (-b \times$ ADC), where $S_{\mathrm{b}}$ and $S_{0}$ are the signal intensity acquired with the diffusion gradient factors of $b$ and 0 , respectively. In this study, MR signal intensities of $b=0$ and $1000 \mathrm{~s} / \mathrm{mm}^{2}$ were used to calculate the ADC. ${ }^{23}$ DWI processing was performed on an off-line workstation (Verio; Siemens). The IVIM model is mathematically expressed with the following equation ${ }^{13,14}$ :

1) $S_{\mathrm{b}} / S_{0}=(1-f) \times \exp (-b D)+f \times \exp \left[-b\left(D+D^{\star}\right)\right]$,

where $S_{\mathrm{b}}$ and $S_{0}$ are the signal intensities in the diffusion gradient factors of $b$ and 0 , respectively, ${ }^{17,18} f$ is the perfusion fraction related to microcirculation, $D$ is the diffusion coefficient representing pure molecular diffusion (the slow component of diffusion), and $D^{*}$ is the pseudodiffusion coefficient representing incoherent microcirculation within the voxel (perfusion-related diffusion or the fast component of diffusion). Three parameters, $D, D^{*}$, and $f$, can be derived from IVIM by fitting the biexponential curve using a nonlinear least-squares technique. Because $D^{*}$ is notably greater than $D$, its influence on signal decay can be neglected when the b-value is $>200 \mathrm{~s} / \mathrm{mm}^{2}$. In that case, Equation 1 can be simplified and the estimation of $D$ can be obtained using only b-values of $>200 \mathrm{~s} / \mathrm{mm}^{2}$, with a simple linear fitting Equation 2:

2)

$$
S_{\mathrm{b}} / S_{0}=\exp (-\mathrm{b} D) .
$$

With the $D$ value determined using Equation 2, $f$ and $D^{*}$ values can be calculated using a nonlinear regression algorithm based on Equation 1. IVIM processing was conducted by using Matlab (Version 7.9; MathWorks, Natick, Massachusetts).

The ADC value derived from conventional DWI, along with $D, D^{*}$, and $f$ values that were derived from IVIM, was measured independently by 2 radiologists (readers 1 and 2 with 10 and 7 years of experience in head and neck imaging, respectively) who were blinded to clinical and histopathologic data. The polygonal ROIs (mean area, $52.93 \pm 37.82 \mathrm{~mm}^{2}$; range, $26-731 \mathrm{~mm}^{2}$ ) were drawn along the outer margin of the lesion on the largest slice of the corresponding parameter maps. Obvious necrotic, cystic, calcification, or hemorrhagic regions were avoided to focus on the solid portion of the tumor as much as possible by referring to T2-weighted and contrast-enhanced T1-weighted images. The measurements made by readers 1 and 2 were used to evaluate the interreader reproducibility. To evaluate intrareader reproducibility, these measurements were repeated by reader 1 , with a minimum washout period of at least 1 month. The average of the 3 measurement results of readers 1 and 2 was used for the statistical analysis.

\section{Statistical Analysis}

All ADC and IVIM parameters of sinonasal lesions are presented as mean $\pm \mathrm{SD}$. The inter- and intrareader reproducibility for ADC and IVIM parameter measurements were evaluated using the intraclass correlation coefficient with $95 \%$ confidence intervals. An intraclass correlation coefficient of $>0.75$ was considered indicative of good agreement.

A simple linear regression analysis was performed to calculate the Pearson correlation coefficient between the patients' ages and conventional DWI and IVIM parameters of the individual lesions. A correlation coefficient $(r)$ of $0.75-1.00$ indicated very good-to-excellent correlation; $0.50-0.74$, moderate-togood correlation; $0.25-0.49$, fair correlation; and $\leq 0.24$, little or no correlation.

The mean $\mathrm{ADC}, D, D^{*}$, and $f$ values were compared between benign and malignant sinonasal lesions using the Student $t$ test. The receiver operating characteristic (ROC) analysis curves with 10 -fold cross-validation were obtained to determine the optimal cutoff value for significant parameters for differentiating benign and malignant sinonasal lesions. The multivariate logistic regression models, including 2 or 3 of these significant parameters, were 


\begin{tabular}{llc}
\hline \multicolumn{1}{c}{ Parameters } & Benign Lesions $(\boldsymbol{n}=\mathbf{5 6})$ & Malignant Lesions $(\boldsymbol{n}=\mathbf{7 5})$ \\
\hline Mean age (yr) & $43.86 \pm 14.11$ & $52.27 \pm 15.21$ \\
Sex (female/male) & $24: 32$ & $24: 51$ \\
Histologic subtypes & Inflammatory polyps (28) & Squamous cell carcinoma (23) \\
& Inverted papilloma (14) & Olfactory neuroblastoma (13) \\
& Fibroangioma (5) & Malignant melanoma (12) \\
& Spindle cell tumor (4) & Rhabdomyosarcoma (9) \\
& Schwannoma (2) (2) & Lymphoma (6) \\
& Ossifying fibroma (2) & Adenoid cystic carcinoma (5) \\
& Enamel cell tumor (1) & Undifferentiated carcinoma (2) \\
& & Osteosarcoma (2) \\
& & Neuroendocrine carcinoma (2) \\
& & Malignant fibrohistiocytoma (1) \\
\hline
\end{tabular}

${ }^{\text {a }}$ Data in parentheses indicate the number of corresponding patients. All inverted papillomas did not have the potential for association with/conversion to squamous cell carcinoma, proven by histopathology.
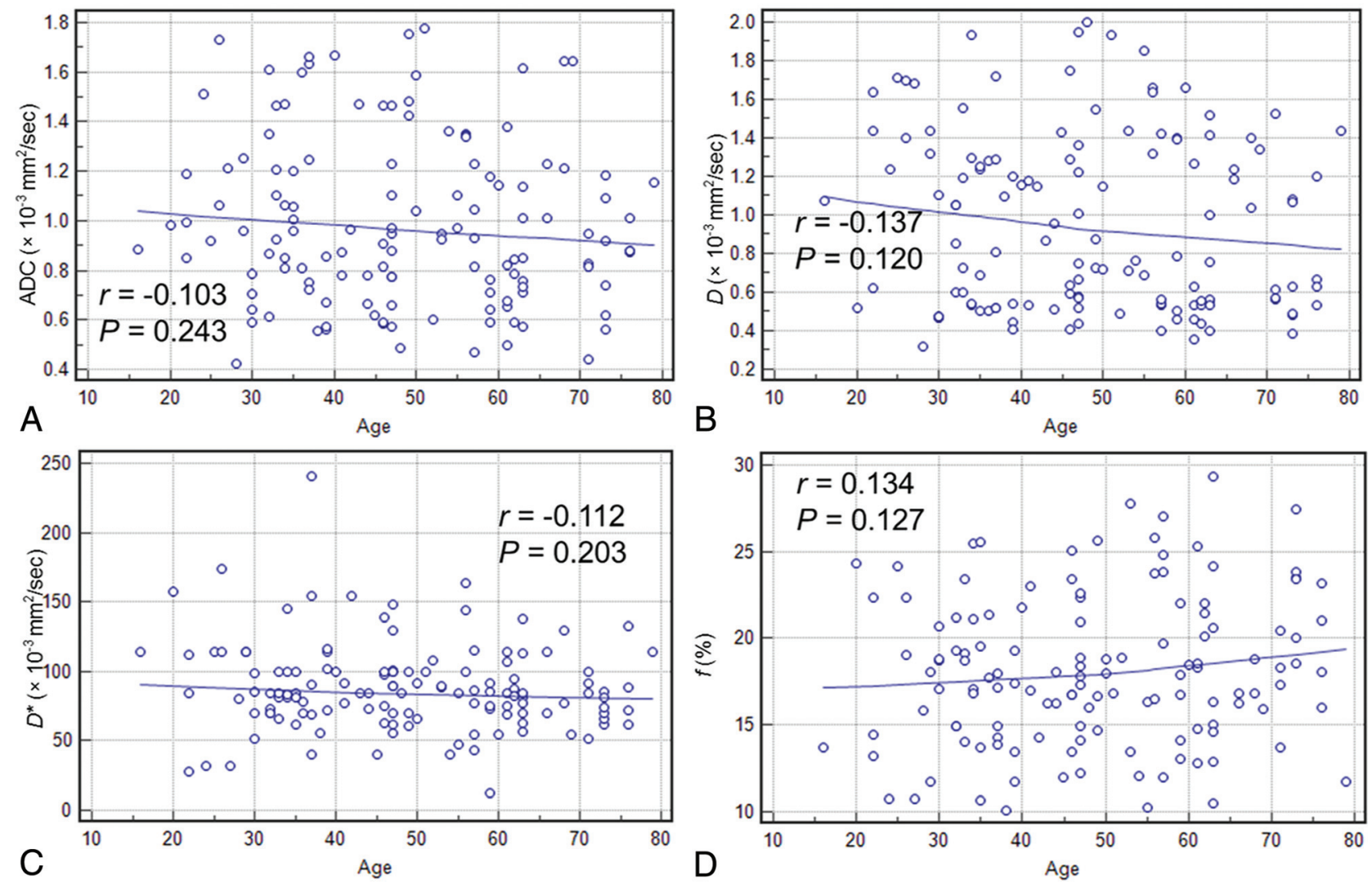

FIG 1. Scatterplots depicting the correlations between the patients' ages and ADC values $(A)$, the patients' ages and $D$ values ( $B)$, the patients' ages and $D^{*}$ values $(C)$, and the patients' ages and $f$ values $(D)$ for all sinonasal lesions.

generated. For each logistic regression model, cross-validation was performed with a 10 -fold procedure. The optimal cutoff value was determined as the point in the upper left-hand corner that maximized the sum of the sensitivity and specificity. The area under the curve (AUC), sensitivity, specificity, accuracy, positive predictive value, and negative predictive value of the single and combined imaging parameters for differential diagnosis were calculated. A Bonferroni correction was used to correct for multiple comparisons.

Statistical analyses were performed in Excel 2013 (Microsoft, Redmond, Washington), MedCalc statistical software (Version 15.2.2; MedCalc Software, Mariakerke, Belgium), and R, Version R 2.15.3 (R Project for Statistical Computing, http://www.r-project.org). ${ }^{24} P$ values $<.05$ were considered significant.

\section{RESULTS}

A total of 131 patients, including 56 patients with benign sinonasal lesions and 75 with malignant sinonasal lesions, were enrolled in this study. Of these patients, 48 were female and 83 were male. The age of patients ranged from 16 to 79 years, with a mean age of 48.67 years. The demographics and histology of benign and malignant sinonasal lesions are summarized in Table 1. Poor correlations were found between the patients' ages and conventional DWI and IVIM parameters, with $r$ ranging from -0.103 to 0.137 (all $P>.05$, Fig 1) 
Table 2: Inter- and intrareader reproducibility for ADC, $D, D^{*}$, and f measurements ${ }^{a}$

\begin{tabular}{lll}
\hline \multirow{2}{*}{\multicolumn{1}{c}{ Parameters }} & \multicolumn{2}{c}{ ICC } \\
\cline { 2 - 3 } & \multicolumn{1}{c}{ Interreader } & \multicolumn{1}{c}{ Intrareader } \\
\hline $\mathrm{ADC}\left(\times 10^{-3} \mathrm{~mm}^{2} / \mathrm{s}\right)$ & $0.961(0.913-0.977)$ & $0.954(0.871-0.980)$ \\
$D\left(\times 10^{-3} \mathrm{~mm}^{2} / \mathrm{s}\right)$ & $0.942(0.860-0.986)$ & $0.936(0.884-0.975)$ \\
$D^{*}\left(\times 10^{-3} \mathrm{~mm}^{2} / \mathrm{s}\right)$ & $0.840(0.762-0.931)$ & $0.848(0.766-0.909)$ \\
$f(\%)$ & $0.908(0.819-0.965)$ & $0.922(0.835-0.964)$ \\
\hline
\end{tabular}

Note:-ICC indicates intraclass correlation coefficient.

${ }^{a}$ Data in parentheses are $95 \%$ confidence intervals.

As shown in Table 2, excellent inter- and intrareader agreement were achieved in the measurement of ADC and IVIM parameters. The mean $\mathrm{ADC}, D, D^{*}$, and $f$ values of benign and malignant sinonasal lesions are shown in Fig 2 and Table 3. The mean $\mathrm{ADC}$ and $D$ values were significantly lower in malignant sinonasal lesions than in benign sinonasal lesions (both $P<.001$ ). The mean $f$ value of malignant sinonasal masses was higher than that of benign sinonasal masses $(P=.001)$. There was no significant difference in the mean $D^{\star}$ value between the 2 groups $(P>.05)$ (Figs 3 and 4).

The diagnostic performance of $\mathrm{ADC}, D$, and $f$ values for differentiation of benign and malignant sinonasal lesions is demonstrated in Table 4 and Fig 5. From the ROC analysis with 10-fold cross-validation, the largest cross-validated AUC was obtained for $D$ compared with $\mathrm{ADC}$ and $f$ (AUC for $D$ versus $\mathrm{ADC}=$ 0.899 versus 0.735 , corrected $P=.009$; AUC for $D$ versus $f=0.899$ versus 0.656 , corrected $P<.0001$; AUC for $\operatorname{ADC}$ versus $f=0.735$ versus 0.656 , corrected $P=.08$ ) in the discrimination of benign and malignant sinonasal lesions. No significant differences were found in the cross-validated AUC among different multiparametric models, including $\mathrm{ADC}+D, \mathrm{ADC}+f, D+f, \mathrm{ADC}+D+f$ (all corrected $P>.05$ ), and the highest AUC was obtained for the model of $\mathrm{ADC}+D+f$, followed by the models of $D+f$, $\mathrm{ADC}+D$, and $\mathrm{ADC}+f$. Compared with single-parametric models (except $D$ ), multiparametric models can significantly improve the cross-validated AUCs for the differentiation of benign and malignant sinonasal lesions (all corrected $P<.05$ ). Additionally, the combination of $D$ and $f$ values derived from IVIM can provide a better diagnostic performance than the ADC value (corrected $P<.001)$.

\section{DISCUSSION}

Our findings showed that quantitative analysis of conventional DWI and IVIM parameters could be helpful in discriminating benign and malignant sinonasal lesions. Additionally, compared with the ADC value derived from conventional DWI, IVIM parameters (especially the combination of $D$ and $f$ values) can significantly improve the diagnostic performance for differentiating solid sinonasal lesions.

The ADC value quantitating free-water molecule motion within a tissue correlates with tumor cellularity. ${ }^{15,23}$ In the present study, the mean ADC value of malignant sinonasal lesions was significantly lower than that of benign lesions; this finding was in accordance with the results from previous studies. ${ }^{9-12,25}$ Histopathologically, malignancies often exhibit high cellularity, effectively restricting the water molecule diffusion of tumors. ${ }^{25}$ Moreover, the present patient cohort included 44 patients with small round blue cell tumors, including lymphoma, undifferentiated carcinoma, malignant melanoma, neuroendocrine carcinoma, olfactory neuroblastoma, rhabdomyosarcoma, and so forth. This heterogeneous group of malignant neoplasms shares common histologic characteristics, including a relatively small cell volume, deep nuclear staining, and high tumor nucleus/cytoplasm ratios, resulting in significantly low ADC values. ${ }^{26}$ Hence, our study revealed that ADC could be a useful imaging parameter for the differentiation of benign and malignant sinonasal lesions. Nevertheless, ADC is derived from conventional DWI with an assumption that the water molecule diffusion is a random motion that follows Gaussian distribution and is calculated by a simple monoexponential decay fitting with $2 \mathrm{~b}$-values. In fact, the measured diffusion signals in living tissues were not simply influenced by the motion of water molecules but also by the perfusion of blood microcirculation at low b-values. Therefore, ADC values obtained from conventional DWI do not exclusively correspond to molecular diffusivity. ${ }^{27,28}$ The IVIM model was recommended to account for molecular diffusion driven by Brownian motion and perfusion-related pseudodiffusion.

IVIM with a biexponential model is a method initially proposed by Le Bihan et $\mathrm{al}^{13,14}$ to quantitatively assess the microscopic translational motion that occurs in each image voxel on MR imaging and to distinguish both pure molecular diffusion and capillary perfusion with sufficiently low b-values $(<200$ $\mathrm{s} / \mathrm{mm}^{2}$ ). However, the value of IVIM in differentiating benign and malignant sinonasal lesions has not been investigated previously, to our knowledge. Until now, 2 main calculation methods, including a conventional method using a nonlinear, biexponential fitting and a newly simplified method using a linear fitting of a logarithmic transformation, have been applied to produce the final IVIM results. ${ }^{29,30}$

Recently, Sasaki et $\mathrm{al}^{30}$ reported that the simplified technique could be an alternative to the conventional method in the determination of IVIM parameters for head and neck tumors. Nonetheless, the conventional calculation method could better predict lesion perfusion and diffusion characteristics with fewer artifacts and higher signal-to-noise ratios compared with the simplified technique. ${ }^{30}$ In addition, the conventional calculation technique using the 2-step fitting with $D$ value estimation first was found to best correlate with dynamic contrast-enhanced MR imaging. ${ }^{29}$ Thus, the conventional method with 2-step fitting was adopted to calculate the IVIM parameters for the differentiation of sinonasal lesions. In this preliminary study, we found that IVIM was a more effective tool than conventional DWI in the differentiation of benign and malignant sinonasal lesions. Additionally, the reproducibility of $D$, $D^{*}$, and $f$ values derived from IVIM was excellent; this finding was consistent with that of Jia et al, ${ }^{18}$ suggesting that IVIM could also be a reliable method for discerning benign from malignant lesions in the sinonasal area.

Our study showed that the $D$ value was significantly lower in malignant sinonasal tumors than in benign lesions. Sumi et $\mathrm{al}^{22}$ reported that the $D$ values of malignant salivary gland tumors were significantly different from those of benign tumors. Similar findings were also reported in other malignan- 

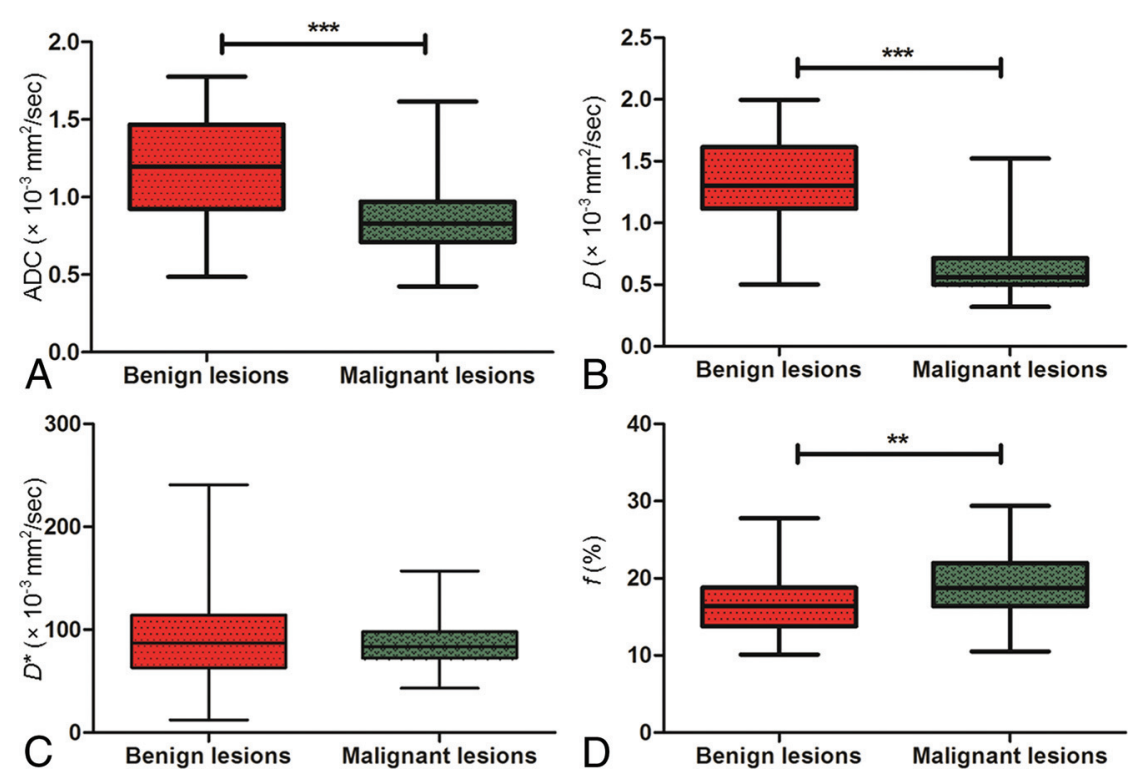

FIG 2. Comparisons of the mean $A D C(A), D(B), D^{*}(C)$, and $f(D)$ values between benign and malignant sinonasal lesions using the Student $t$ test. Triple asterisks indicate $P<.001$; double asterisks, $P<.01$.

Table 3: Comparisons of ADC, $D, D^{*}$, and $f$ values between benign and malignant sinonasal lesions ${ }^{2}$

\begin{tabular}{lccr}
\multicolumn{1}{c}{ Parameters } & $\begin{array}{c}\text { Benign Lesions } \\
(\boldsymbol{n}=\mathbf{5 6})\end{array}$ & $\begin{array}{c}\text { Malignant Lesions } \\
(\boldsymbol{n}=\mathbf{7 5})\end{array}$ & $\begin{array}{c}\boldsymbol{P} \\
\text { Value }\end{array}$ \\
\hline $\mathrm{ADC}\left(\times 10^{-3} \mathrm{~mm}^{2} / \mathrm{s}\right)$ & $1.163 \pm 0.354$ & $0.862 \pm 0.258$ & $<.001$ \\
$D\left(\times 10^{-3} \mathrm{~mm}^{2} / \mathrm{s}\right)$ & $1.322 \pm 0.347$ & $0.677 \pm 0.299$ & $<.001$ \\
$D^{*}\left(\times 10^{-3} \mathrm{~mm}^{2} / \mathrm{s}\right)$ & $90.470 \pm 40.756$ & $86.445 \pm 22.865$ & .474 \\
$f(\%)$ & $16.656 \pm 4.274$ & $19.211 \pm 4.066$ & .001 \\
\hline
\end{tabular}

${ }^{a}$ Except for the $P$ values, data are expressed as the mean \pm SD.

cies in other body organs, such as the breast ${ }^{31}$ and prostate. ${ }^{32}$ These findings may be explained by hypercellularity in malignant lesions that reduced the microstructure diffusion space of water molecules, resulting in a decrease in the $D$ value. Hence, the $D$ value can be highly valuable for differentiating benign and malignant sinonasal lesions. $D^{\star}$ and $f$ are perfusion-related parameters derived from IVIM, which are significantly correlated with microvessel density, a surrogate marker of angiogenesis. ${ }^{33}$ The $D^{*}$ value is determined as the signal intensity ratios of blood capillaries and is considered linked to perfusion, which, in turn, may be dependent on tumor microvessel density. ${ }^{13}$ Additionally, $D^{*}$ was reported to be proportional to the mean capillary segment length and average blood velocity; thus, it is reflective of tumor vascularity. ${ }^{13}$ In our present study, we found no significant difference in the $D^{*}$ value between benign and malignant sinonasal lesions, which was in satisfactory agreement with previous studies. ${ }^{34,35}$ However, our $D^{*}$ estimation in sinonasal lesions was not consistent with those reported by Kim et $\mathrm{al}^{36}$ for pancreatic pathologies and Sumi et $\mathrm{al}^{22}$ for salivary gland tumors, which may be due to the low signal-to-noise ratio and the relatively poor measurement reproducibility of $D^{*} .{ }^{31}$ As for the $f$ value, it measures the fractional volume of capillary blood flowing in each voxel and has direct correlations with the extent of normal angiogenesis with intact vessels in terms of basement membrane thickness and pericyte coverage. ${ }^{37,38}$ Therefore, $f$ may be an indicator of vascular permeability. In the current study, we found that the $f$ value was significantly higher in malignant lesions than in benign sinonasal lesions, a finding in accordance with those in previous studies on head and neck tumors. ${ }^{17,19,22,30}$ With the application of dynamic contrast-enhanced imaging, Xian et $\mathrm{al}^{39}$ found that malignant sinonasal tumors were usually associated with high permeability and high perfusion, which was in good agreement with our results. However, some malignant sinonasal lesions, such as lymphomas, can have a low $f$ value because of low levels of vessel density within scarce amounts of stromal tissue. ${ }^{17}$ Moreover, some benign sinonasal lesions can also be associated with a high perfusion level. For example, small vessels with ectasia are frequently observed in the Antoni $\mathrm{B}$ areas of schwannomas, resulting in a high $f$ value. ${ }^{17}$

Three parameters, $\mathrm{ADC}, D$, and $f$, were significant for differentiating benign from malignant sinonasal lesions. The ROC analysis with 10-fold cross-validation showed that the cross-validated AUC, sensitivity, specificity, and accuracy levels of the $D$ value were significantly higher than those of the $f$ and ADC values. As previously reported, cellularity and microcirculation influence ADC measurements in a diametrically opposite direction. ${ }^{40}$ However, most malignant tumors had both hypercellularity (low diffusivity) and hypervascularity (high perfusion fraction). $D$, known as the pure molecular diffusion coefficient, can represent cellularity more precisely, can reduce the bias by avoiding microcirculation contributions, ${ }^{13,14}$ and, therefore, can better differentiate benign and malignant sinonasal lesions. Additionally, the combination of $D$ and $f$ values derived from IVIM can provide a better diagnostic performance than the ADC value, indicating that IVIM could be a more efficient method than conventional DWI for the differentiation of sinonasal lesions.

There are some limitations to our study. First, given the wide variety of histologic types and the small number of each type of benign and malignant sinonasal lesion, selection bias was inevitable, so our findings still need larger prospective studies to further validate them. Additionally, as in previous studies, ${ }^{11,12}$ inflammatory polyps were included in our study. These typically are not a 

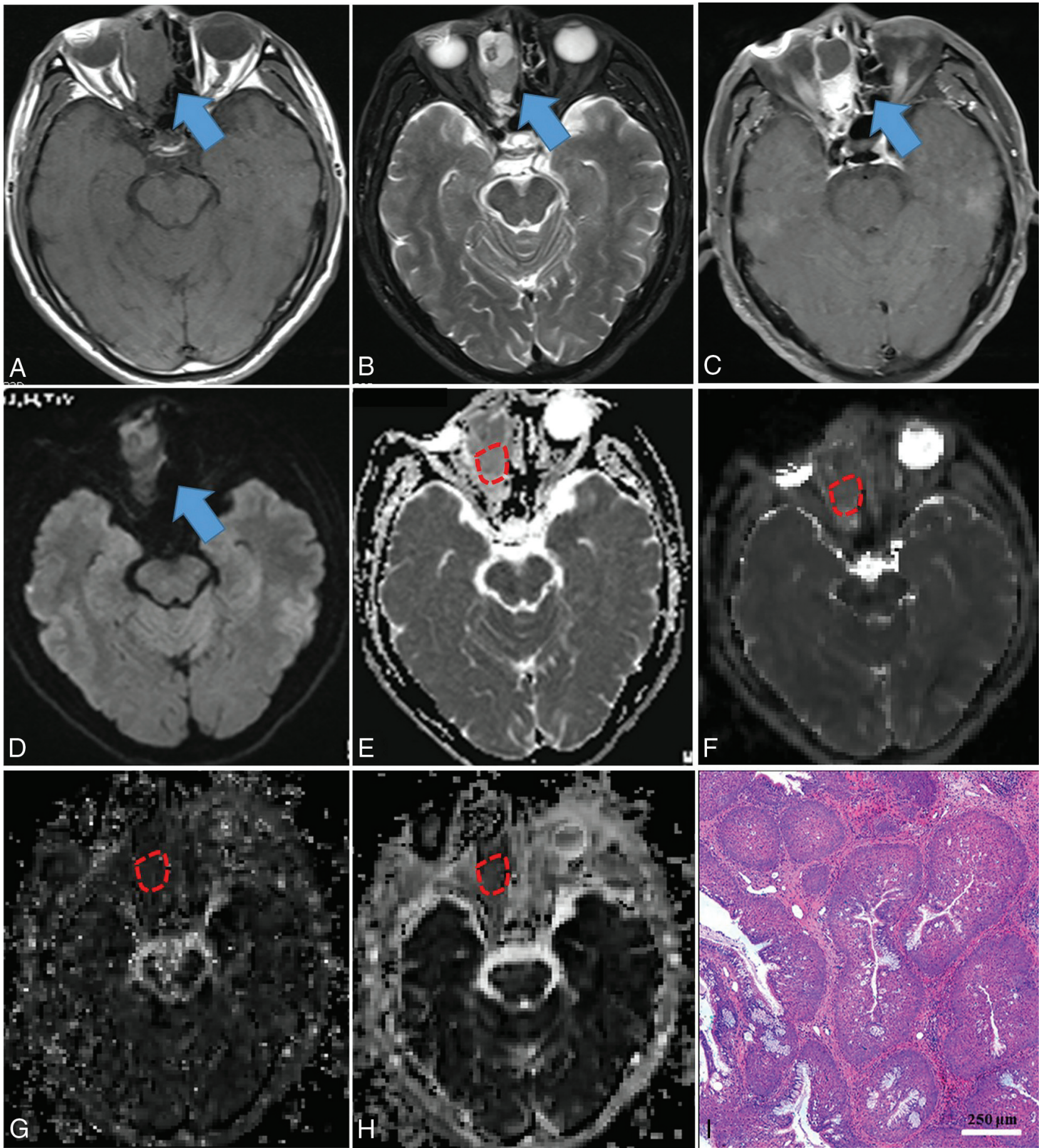

FIG 3. Inverted papilloma in a 71-year-old man. A mass was predominantly located in the right ethmoid sinus with involvement of the right nasal cavity (blue arrow), demonstrating heterogeneous hypo- (necrosis) to isointensity (tumor cells) on TIWI (A), iso- (tumor cells) to hyperintensity (necrosis) on T2WI (B), and heterogeneously intense enhancement on contrast-enhanced TIWI (C), compared with normal-appearing gray matter. The mass showed isointensity on the DWI (blue arrow) $(D)$ compared with normal-appearing gray matter. On the ADC map (E), the mass showed a slightly high signal intensity with an ADC value of $1.425 \times 10^{-3} \mathrm{~mm}^{2} / \mathrm{s}$ (red polygonal ROI). On IVIM images, the mass appeared obviously iso- to hyperintense on the $D$ map $(F)$ with a $D$ value of $0.871 \times 10^{-3} \mathrm{~mm}^{2} / \mathrm{s}$ and appeared isointense on the $D^{*}(G)$ and $f$ maps $(H)$ with $D^{*}$ and $f$ values of $61.048 \times 10^{-3} \mathrm{~mm}^{2} / \mathrm{s}$ and $25.651 \%$, respectively (red polygonal ROIs). Hematoxylin-eosin staining (I) confirmed the mass as an inverted papilloma (original magnification, $\times 100$; scale bar, $250 \mu \mathrm{m}$ ).

diagnostic dilemma because they usually do not demonstrate solid masslike enhancement, bone change, or masquerade as tumors. However, most inflammatory polyps included in our study were hemorrhagic and/or necrotic polyps (19/28), which sometimes may be difficult to discriminate from malignant sinonasal lesions on conventional CT and MR imaging. Second, the ROI placement for the measurement of conventional DWI and IVIM parameters could not be correlated with fragmental histologic specimens on a site-to-site basis. Third, due to the artifacts from air in the sinuses, there may be some distortions in the measured 

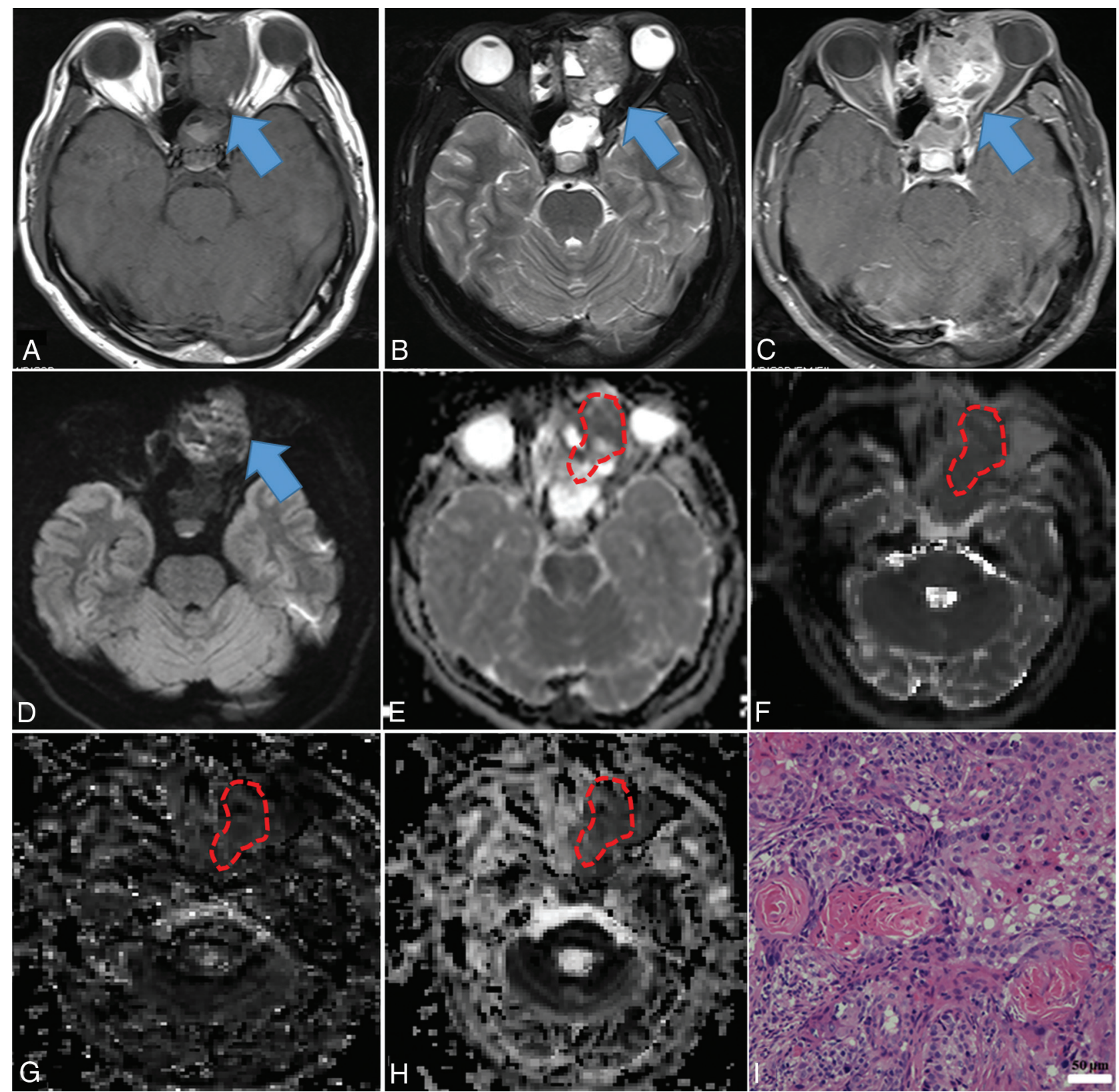

FIG 4. Squamous cell carcinoma in a 53-year-old man. A mass was predominantly located in the left ethmoid sinus with involvement of the ipsilateral orbit, nasal cavity, and sphenoid sinus (blue arrow), demonstrating heterogeneous hypo- (necrosis) to isointensity (tumor cells) on TIWI (A), iso- (tumor cells) to hyperintensity (necrosis) on T2WI (B), and heterogeneously intense enhancement on contrast-enhanced TIWI (C), compared with normal-appearing gray matter. The mass showed heterogeneously hypo- to hyperintensity on the DWI (blue arrow) (D) compared with normal-appearing gray matter. On the ADC map $(E)$, the mass showed hypointensity with an $A D C$ value of $0.872 \times 10^{-3} \mathrm{~mm}^{2} / \mathrm{s}$ (red polygonal ROI). On IVIM images, the mass appeared dramatically hypointense on the $D$ map $(F)$, with a $D$ value of $0.533 \times 10^{-3} \mathrm{~mm}^{2} / \mathrm{s}$, hypoto isointense on the $D^{*} \operatorname{map}(G)$, and iso- to hyperintense on the $f$ map $(H)$, with $D^{*}$ and $f$ values of $77.473 \times 10^{-3} \mathrm{~mm}^{2} / \mathrm{s}$ and $22.966 \%$, respectively (red polygonal ROIs). Hematoxylin-eosin staining (I) confirmed the mass as a squamous cell carcinoma (original magnification, $\times 200 ;$ scale bar, $50 \mu \mathrm{m})$.

volume that may result in measurement bias. Thus, conventional DWI and IVIM data acquired using readout-segmented echoplanar imaging using parallel imaging and 2D navigator-based reacquisition may be helpful to reduce the artifacts and distortions. ${ }^{12,41}$ Fourth, the value of all these parameters, including ADC, $D, D^{*}$, and $f$, in the differentiations of pathologic types of sinonasal tumors was not evaluated because of the limited number of each type of sinonasal lesion. Finally, because IVIM parameters could be affected by the calculation method, the threshold values proposed in our study may not be generalizable to other institutions or other methods of IVIM calculation. This issue needs to be investigated in further studies.

\section{CONCLUSIONS}

Our preliminary study shows that the ADC value from conventional DWI and the $D$ and $f$ values from IVIM may be helpful for discriminating markers in the differentiation of benign and malignant sinonasal lesions. Furthermore, the combination of 
Table 4: Diagnostic performance of single parameters ( $A D C, D$ and $f$ ) and combined parameters (ADC $+f, A D C+D, D+f$, and $A D C+D+f$ ) for the differentiation of benign and malignant sinonasal lesions using receiver operating characteristic curve analysis with 10 -fold cross-validation

\begin{tabular}{lcccccccc}
\hline & TV & AUC & Cross-Validated AUC & Sensitivity & Specificity & Accuracy & Precision & F Score \\
\hline ADC & 0.919 & 0.754 & 0.735 & 0.800 & 0.547 & 0.686 & 0.684 & 0.738 \\
$D$ & 0.715 & 0.907 & 0.899 & 0.841 & 0.816 & 0.831 & 0.866 & 0.853 \\
$f$ & 16.995 & 0.676 & 0.656 & 0.723 & 0.472 & 0.610 & 0.627 & 0.723 \\
ADC $+f$ & - & 0.910 & 0.907 & 0.857 & 0.792 & 0.831 & 0.857 & 0.857 \\
$A D C+D$ & - & 0.944 & 0.914 & 0.813 & 0.778 & 0.797 & 0.813 & 0.813 \\
$D+f$ & - & 0.938 & 0.935 & 0.914 & 0.813 & 0.873 & 0.877 & 0.895 \\
ADC $+D+f$ & - & 0.951 & 0.944 & 0.900 & 0.833 & 0.873 & 0.887 & 0.894 \\
\hline
\end{tabular}

Note:-TV indicates threshold value.

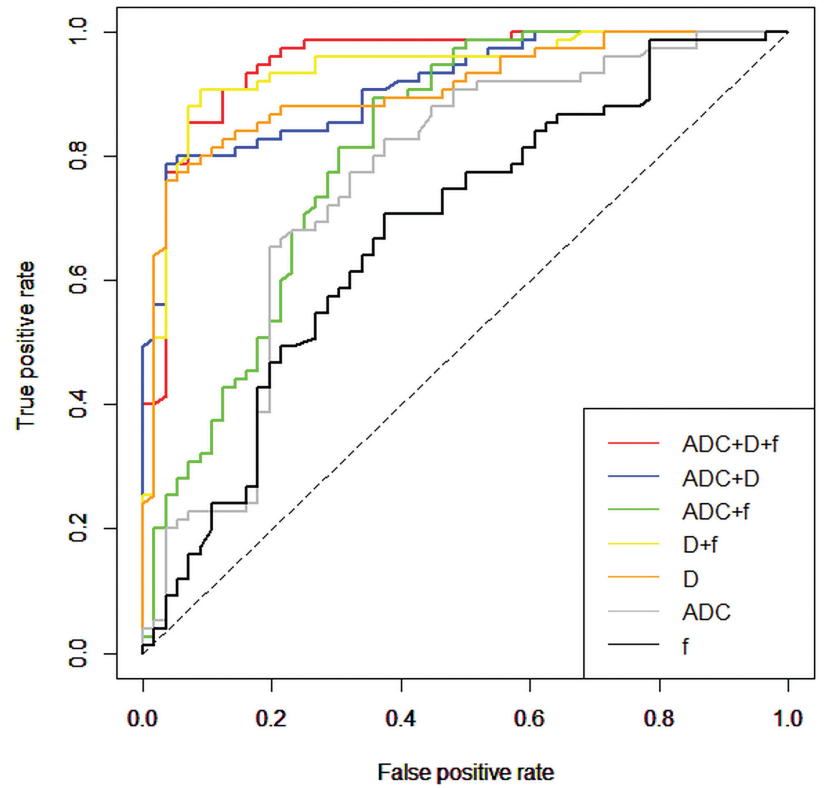

FIG 5. ROC curves with 10 -fold cross-validation of single- parameter models (including $A D C, D$, and $f$ ) and multiparametric models (including $A D C+f, A D C+D, D+f$, and $A D C+D+f$ ) in the contrast-enhancing lesions for differentiating benign from malignant sinonasal lesions.

$D$ and $f$ values demonstrates significantly higher sensitivity, specificity, and accuracy than the ADC value, revealing that IVIM appears to be a more valuable tool than conventional DWI for distinguishing benign from malignant sinonasal lesions. Further prospective studies are needed to confirm these findings.

\section{REFERENCES}

1. Slootweg PJ, Ferlito A, Cardesa A, et al. Sinonasal tumors: a clinicopathologic update of selected tumors. Eur Arch Otorhinolaryngol 2013;270:5-20 CrossRef Medline

2. Su SY, Kupferman ME, DeMonte F, et al. Endoscopic resection of sinonasal cancers. Curr Oncol Rep 2014;16:369 CrossRef Medline

3. Eggesbø HB. Imaging of sinonasal tumours. Cancer Imaging 2012; 12:136-52 CrossRef Medline

4. Koeller KK. Radiologic features of sinonasal tumors. Head Neck Pathol 2016;10:1-12 CrossRef Medline

5. Wang XY, Yan F, Hao H, et al. Improved performance in differentiating benign from malignant sinonasal tumors using diffusionweighted combined with dynamic contrast-enhanced magnetic resonance imaging. Chin Med J (Engl) 2015;128:586-92 CrossRef Medline

6. Jégoux F, Metreau A, Louvel G, et al. Paranasal sinus cancer. Eur Ann Otorhinolaryngol Head Neck Dis 2013;130:327-35 CrossRef Medline

7. Carta F, Blancal JP, Verillaud B, et al. Surgical management of in- verted papilloma: approaching a new standard for surgery. Head Neck 2013;35:1415-20 CrossRef Medline

8. Madani G, Beale TJ, Lund VJ. Imaging of sinonasal tumors. Semin Ultrasound CT MR 2009;30:25-38 CrossRef Medline

9. Sasaki M, Eida S, Sumi M, et al. Apparent diffusion coefficient mapping for sinonasal diseases: differentiation of benign and malignant lesions. AJNR Am J Neuroradiol 2011;32:1100-06 CrossRef Medline

10. Sasaki M, Sumi M, Eida S, et al. Multiparametric MR imaging of sinonasal diseases: time-signal intensity curve- and apparent diffusion coefficient-based differentiation between benign and malignant lesions. AJNR Am J Neuroradiol 2011;32:2154-59 CrossRef Medline

11. White ML, Zhang Y, Robinson RA. Evaluating tumors and tumorlike lesions of the nasal cavity, the paranasal sinuses, and the adjacent skull base with diffusion-weighted MRI. J Comput Assist Tomogr 2006;30:490-95 CrossRef Medline

12. Wang F, Sha Y, Zhao M, et al. High-resolution diffusion-weighted imaging improves the diagnostic accuracy of dynamic contrast-enhanced sinonasal magnetic resonance imaging. J Comput Assist Tomogr 2017;41:199-205 CrossRef Medline

13. Le Bihan D. Intravoxel incoherent motion imaging using steadystate free precession. Magn Reson Med 1988;7:346-51 CrossRef Medline

14. Le Bihan $\mathrm{D}$, Breton $\mathrm{E}$, Lallemand $\mathrm{D}$, et al. MR imaging of intravoxel incoherent motions: application to diffusion and perfusion in neurologic disorders. Radiology 1986;161:401-07 CrossRef Medline

15. Iima M, Le Bihan D. Clinical intravoxel incoherent motion and diffusion MR imaging: past, present, and future. Radiology 2016;278: 13-32 CrossRef Medline

16. Fujima N, Yoshida D, Sakashita T, et al. Intravoxel incoherent motion diffusion-weighted imaging in head and neck squamous cell carcinoma: assessment of perfusion-related parameters compared to dynamic contrast-enhanced MRI. Magn Reson Imaging 2014;32: 1206-13 CrossRef Medline

17. Sumi M, Nakamura T. Head and neck tumors: assessment of perfusion-related parameters and diffusion coefficients based on the intravoxel incoherent motion model. AJNR Am J Neuroradiol 2013;34: 410-16 CrossRef Medline

18. Jia QJ, Zhang SX, Chen WB, et al. Initial experience of correlating parameters of intravoxel incoherent motion and dynamic contrastenhanced magnetic resonance imaging at $3.0 \mathrm{~T}$ in nasopharyngeal carcinoma. Eur Radiol 2014;24:3076-87 CrossRef Medline

19. Lai V, Li X, Lee VH, et al. Nasopharyngeal carcinoma: comparison of diffusion and perfusion characteristics between different tumour stages using intravoxel incoherent motion MR imaging. Eur Radiol 2014;24:176-83 CrossRef Medline

20. Hauser T, Essig M, Jensen A, et al. Prediction of treatment response in head and neck carcinomas using IVIM-DWI: evaluation of lymph node metastasis. Eur J Radiol 2014;83:783-87 CrossRef Medline

21. Fujima N, Yoshida D, Sakashita T, et al. Prediction of the treatment outcome using intravoxel incoherent motion and diffusional kurtosis imaging in nasal or sinonasal squamous cell carcinoma patients. Eur Radiol 2017;27:956-65 CrossRef Medline 
22. Sumi M, Van Cauteren M, Sumi T, et al. Salivary gland tumors: use of intravoxel incoherent motion MR imaging for assessment of diffusion and perfusion for the differentiation of benign from malignant tumors. Radiology 2012;263:770-77 CrossRef Medline

23. Le Bihan D. Molecular diffusion, tissue microdynamics and microstructure. NMR Biomed 1995;8:375-86 CrossRef Medline

24. Jung Y, Hu J. A K-fold averaging cross-validation procedure. J Nonparametr Stat 2015;27:167-79 CrossRef Medline

25. Jiang JX, Tang ZH, Zhong YF, et al. Diffusion kurtosis imaging for differentiating between the benign and malignant sinonasal lesions. J Magn Reson Imaging 2017;45:1446-54 CrossRef Medline

26. Bridge JA, Bowen JM, Smith RB. The small round blue cell tumors of the sinonasal area. Head Neck Pathol 2010;4:84-93 CrossRef Medline

27. Thoeny HC, De Keyzer F, Boesch C, et al. Diffusion-weighted imaging of the parotid gland: influence of the choice of $b$-values on the apparent diffusion coefficient value. J Magn Reson Imaging 2004;20: 786-90 CrossRef Medline

28. Padhani AR, Liu G, Koh DM, et al. Diffusion-weighted magnetic resonance imaging as cancer biomarker: consensus and recommendations. Neoplasia 2009;11:102-25 CrossRef Medline

29. Suo S, Lin N, Wang H, et al. Intravoxel incoherent motion diffusionweighted MR imaging of breast cancer at 3.0 Tesla: comparison of different curve-fitting methods. J Magn Reson Imaging 2015;42: 362-70 CrossRef Medline

30. Sasaki M, Sumi M, Eida S, et al. Simple and reliable determination of intravoxel incoherent motion parameters for the differential diagnosis of head and neck tumors. PLoS One 2014;9:e112866 Medline

31. Liu C, Wang $\mathrm{K}$, Chan Q, et al. Intravoxel incoherent motion MR imaging for breast lesions: comparison and correlation with pharmacokinetic evaluation from dynamic contrast-enhanced MR imaging. Eur Radiol 2016;26:3888-98 CrossRef Medline

32. Pang Y, Turkbey B, Bernardo M, et al. Intravoxel incoherent motion MR imaging for prostate cancer: an evaluation of perfusion fraction and diffusion coefficient derived from different b-value combinations. Magn Reson Med 2013;69:553-62 CrossRef Medline
33. Lee HJ, Rha SY, Chung YE, et al. Tumor perfusion-related parameter of diffusion-weighted magnetic resonance imaging: correlation with histological microvessel density. Magn Reson Med 2014;71: 1554-58 CrossRef Medline

34. Wang LL, Lin J, Liu K, et al. Intravoxel incoherent motion diffusionweighted MR imaging in differentiation of lung cancer from obstructive lung consolidation: comparison and correlation with pharmacokinetic analysis from dynamic contrast-enhanced MR imaging. Eur Radiol 2014;24:1914-22 CrossRef Medline

35. Iima M, Yano K, Kataoka M, et al. Quantitative non-Gaussian diffusion and intravoxel incoherent motion magnetic resonance imaging: differentiation of malignant and benign breast lesions. Invest Radiol 2015;50:205-11 CrossRef Medline

36. Kim B, Lee SS, Sung YS, et al. Intravoxel incoherent motion diffusion-weighted imaging of the pancreas: characterization of benign and malignant pancreatic pathologies. J Magn Reson Imaging 2017; 45:260-69 CrossRef Medline

37. Le Bihan D, Turner R. The capillary network: a link between IVIM and classical perfusion. Magn Reson Med 1992;27:171-78 CrossRef Medline

38. Lewin M, Fartoux L, Vignaud A, et al. The diffusion-weighted imaging perfusion fraction $\mathrm{f}$ is a potential marker of sorafenib treatment in advanced hepatocellular carcinoma: a pilot study. Eur Radiol 2011;21:281-90 CrossRef Medline

39. Xian J, Du H, Wang X, et al. Feasibility and value of quantitative dynamic contrast enhancement MR imaging in the evaluation of sinonasal tumors. Chin Med J (Engl) 2014;127:2259-64 Medline

40. Liu C, Liang C, Liu Z, et al. Intravoxel incoherent motion (IVIM) in evaluation of breast lesions: comparison with conventional DWI. Eur J Radiol 2013;82:e782-89 CrossRef Medline

41. Lu P, Sha Y, Wan H, et al. Assessment of nonarteritic anterior ischemic optic neuropathy with intravoxel incoherent motion diffusion-weighted imaging using readout-segmented echo-planar imaging, parallel imaging, and $2 \mathrm{D}$ navigator-based reacquisition. $J$ Magn Reson Imaging 2017;46:1760-66 CrossRef Medline 